\title{
A Religiosidade/Espiritualidade dos Médicos de Família: Avaliação de Alunos da Universidade Aberta do SUS (UNA-SUS)
}

\author{
The Spirituality/Religiosity of Family Medicine \\ Physicians: Evaluation of SUS Open University \\ (UNA-SUS) Trainees
}

Paulo Rogerio Aguiarl,II Silvio César Cazella ${ }^{I}$ Marcia Rosa Costa

\section{PALAVRAS-CHAVE}

- Espiritualidade.

- Educação Médica.

- Atenção Primária à Saúde.
REVISTA BRASILEIRA DE EDUCAÇÃO MÉDICA
I Universidade Federal de Ciências da Saúde de Porto Alegre, Porto Alegre, RS, Brasil.

${ }^{\text {II }}$ Associação Médico-Espírita do Rio Grande do Sul, Porto Alegre, RS, Brasil. 


\section{KEYWORDS}

- Spirituality.

- Medical Education.

- Primary Health Care.

Recebido em: 13/02/2017

Aprovado em: 27/04/2017

\section{ABSTRACT}

Introduction: Many universities around the world have included religious or spiritual (R/S) issues in the undergraduate medicine curriculum, especially in USA and United Kingdom. The article aims to present the opinion of UNA-SUS/UFCSPA's family medicine trainees about the teaching of R/S in undergraduate medical training, their perceptions about $R / S$ in clinical practice and their personal religious profile. Methodology: Cross-sectional design, using the UNA-SUS/UFCSPA online platform. All Brazilian students were invited to participate. Results: 73 answers were obtained (17.2\% response rate) among 423 students. $61.4 \%$ declared themselves as Catholic, $18.5 \%$ as Protestant and $8.6 \%$ Spiritist; $52.1 \%$ practice no religion, $86.3 \%$ believe in God, $89 \%$ believe that spiritual care is important or very important in health care and $94.6 \%$ consider that spirituality impacts on physical health. $60.3 \%$ consider that spiritual history is the most important ability to be learned and $72.2 \%$ believe that this issue should be taught in the undergraduate curriculum. The relevance attributed to $R / S$ in medical care is not mediated by the student's intrinsic religiosity, because there is no statistical correlation between the variables $(p=0.585)$. Physicians recognize the relevance of spirituality in patients' lives and health, but they are less aware of their role as spiritual care providers. Conclusions: The acceptance of the theme of $R / S$ was considered very satisfactory. It is necessary to develop innovative pedagogic proposals, methodologically suited to medical practice in a public health system, both in graduate and undergraduate programs.

\section{INTRODUÇÃO}

Nas últimas duas décadas, a comunidade científica internacional tem testemunhado um crescente número de pesquisas sobre as relações entre religiosidade/espiritualidade (R/E) e saúde, que apontam um impacto predominantemente positivo em desfechos clínicos em saúde física e mental ${ }^{1,2,3}$. Nesse contexto, a atenção do profissional de saúde para as preocupações religiosas e espirituais dos pacientes tem sido considerada parte de um amplo movimento em direção a uma prática clínica centrada no paciente, culturalmente competente, narrativa e integrati$\mathrm{va}^{4}$. Assim, muitas universidades ao redor do mundo têm incluído o tema R/E no currículo médico no período de graduação, especialmente nos Estados Unidos ${ }^{5}$ e Reino Unido ${ }^{6}$, embora isto ainda não possa ser considerado um fenômeno global ${ }^{7}$.

No Brasil, levantamentos recentes têm indicado opiniões favoráveis de alunos, professores e diretores de faculdades acerca da relevância do tema e sua importância para a formação médica ${ }^{8,9}$. É importante notar que os próprios pacientes desejam, em sua maioria, que o médico esteja aberto ao tema durante a consulta e o inclua na avaliação clínica ${ }^{10}$.

No ensino da pós-graduação, algumas iniciativas também são encontradas na literatura, especialmente nas áreas de cuidados paliativos, psiquiatria e medicina de família, mas, assim como na graduação, faltam estudos mais aprofundados sobre metodologias de ensino e conteúdo das disciplinas ${ }^{7,11,12}$.
Estudos que utilizam metodologias de ensino na modalidade a distância não são encontrados na literatura nacional, e raras experiências de ensino em $\mathrm{R} / \mathrm{E}$ usam essa abordagem ${ }^{13}$.

$\mathrm{O}$ presente estudo integra um projeto de pesquisa que objetiva avaliar uma experiência de ensino de R/E com médicos brasileiros na modalidade de ensino a distância. Assim, um módulo de ensino online em "Espiritualidade na Prática Clínica" foi disponibilizado a médicos atuantes no SUS e que realizavam especialização em Saúde da Família por meio da Universidade Aberta do SUS, na Universidade Federal de Ciências da Saúde de Porto Alegre (UNA-SUS/UFCSPA).

O Sistema Universidade Aberta do SUS (UNA-SUS) do Ministério da Saúde cria condições e possibilita o funcionamento de uma rede colaborativa de instituições públicas de educação superior, conveniadas ao Ministério da Saúde e credenciadas pelo Ministério da Educação, para a oferta de educação a distância destinada a somar esforços em educação permanente dos trabalhadores do SUS ${ }^{14}$. A Rede UNA-SUS, por meio da articulação entre essas instituições, permite maior intercâmbio de experiências e conhecimentos em proveito da melhoria da cooperação para o desenvolvimento de ações educacionais de alcance nacional. Esses profissionais médicos fazem parte de dois programas nacionais: Mais Médicos e Programa de Valorização do Profissional da Atenção Básica (Provab). 
Este artigo ocupa-se exclusivamente da primeira etapa do projeto de pesquisa e objetiva delinear o perfil do médico especializando em Saúde da Família da UNA-SUS/UFCSPA em termos da opinião sobre o ensino do tema religiosidade/ espiritualidade na graduação, suas experiências formativas, as percepções dos profissionais sobre a temática da $R / E$ na prática clínica e os índices de religiosidade desses acadêmicos.

\section{MÉTODOS}

Um instrumento de pesquisa eletrônica foi disponibilizado aos alunos na plataforma virtual da UNA-SUS/UFCSPA, e foram convidados a participar voluntariamente todos os médicos de nacionalidade brasileira inscritos no curso de especialização em Saúde da Família. Foram enviados dois e-mails a esses alunos com um link do formulário de pesquisa entre os meses de junho e agosto de 2016. Médicos de outras nacionalidades inscritos no curso foram excluídos da amostra da pesquisa.

O instrumento foi elaborado para analisar as opiniões dos médicos sobre o tema $\mathrm{R} / \mathrm{E}$ na prática clínica, para verificar experiências prévias de ensino sobre essa temática no período de graduação e para avaliar o perfil de religiosidade dos médicos, informações necessárias à continuidade do projeto em suas etapas subsequentes (elaboração e aplicação do módulo de ensino EAD). Assim, o instrumento de pesquisa foi composto por:

(a) Questionário sociodemográfico, com oito questões;

(b) Questionário de percepções de espiritualidade no cuidado médico, com 13 questões objetivas, sendo sete em escala Likert e uma de múltipla escolha;

(c) Questionário de percepções sobre espiritualidade no ensino médico, com dez questões, sendo duas para respostas abertas, quatro em escala Likert e quatro de múltipla escolha;

(d) Escala da Duke University Religiosity Scale (Durel), com cinco questões objetivas;

(e) Inventário de Religiosidade Intrínseca, com dez questões em escala Likert.

Os questionários de percepções sobre espiritualidade no cuidado médico e no ensino médico foram elaborados para os propósitos desta pesquisa, tendo sido utilizado como referência um questionário do Reino Unido publicado por Bell et al. ${ }^{15}$, que foi traduzido e adaptado. Antes de sua aplicação, houve uma etapa de validação de conteúdo com cinco juízes, experts em medicina de família, que contribuíram para aperfeiçoar o instrumento. As duas escalas de avaliação de religiosidade (Durel ${ }^{14}$ e Inventário de Religiosidade Intrínseca ${ }^{15}$ ) já foram validadas para utilização na população brasileira e fornecem três variáveis de pesquisa: religiosidade intrínseca, religiosidade organizacional e religiosidade não organizacional.
A análise dos dados é apresentada em estatística descritiva simples. Foi considerado um $p<0,05$ como estatisticamente significativo.

Este projeto foi registrado na Plataforma Brasil e aprovado pelo CEP da UFCSPA. A assinatura do Termo de Consentimento Livre e Esclarecido foi solicitada a todos os respondedores desse levantamento.

\section{RESULTADOS}

Foram obtidas 73 respostas $(17,2 \%)$ de um total de 423 médicos brasileiros que frequentavam o curso de especialização em Saúde da Família da UNA-SUS/UFCSPA durante os meses de coleta de dados. A amostra analisada foi de 43 mulheres $(58,9 \%)$ e 30 homens $(41,1 \%)$, com média de idade de 33 anos (DP $=9,1$ anos) e mediana de 2 anos de experiência profissional ( $\mathrm{DP}=8,98$ anos). A maioria dos médicos se autodeclarou de cor branca ( $\mathrm{n}=54,74 \%), 17$ pardos $(23,3 \%), 1$ negro e 1 indígena (1,4\%). Em relação ao estado civil, a maioria dos médicos era solteira $(n=31,42,5 \%)$, sendo 26 casados $(35,6 \%), 12$ morando juntos $(16,4 \%)$ e 4 separados $(5,5 \%)$.

As variáveis relacionadas à $\mathrm{R} / \mathrm{E}$ do médico foram: filiação religiosa (primeira e segunda), crença em Deus, religiosidade organizacional (frequência a templos), religiosidade não organizacional, religiosidade intrínseca e se é praticante. Os resultados são apresentados na Tabela 1.

No questionário de percepções sobre espiritualidade no cuidado médico, avaliou-se a opinião dos médicos a respeito da importância da R/E na prática clínica em comparação a outras dimensões do cuidado assistencial, quais os procedimentos médicos considerados mais relevantes na abordagem do tema e o papel da espiritualidade como integrante no processo de adoecimento e de tratamento dos pacientes. Os resultados são sumariados na Tabela 2.

O questionário de percepções sobre espiritualidade no ensino médico avaliou as experiências formais de aprendizado dos médicos durante o seu período formativo na graduação, bem como opiniões a respeito de como o tema R/E deve ser ensinado, em termos de conteúdo, metodologias e formas de avaliação. Os resultados são apresentados na Tabela 3.

A correlação entre o índice de religiosidade intrínseca, medido pelo Inventário de Religiosidade Intrínseca, e a opinião dos médicos sobre a importância da R/E no cuidado médico é apresentada na Tabela 4.

\section{DISCUSSÃO}

Segundo o conhecimento dos autores, este é o primeiro estudo brasileiro que descreve a R/E de médicos de família, bem como suas experiências e concepções em torno do tema na 


\section{TABELA 1}

Variáveis de R/E dos médicos pesquisados

Filiação religiosa

Católica

Evangélica

$13(18,5)$

Espírita

Agnóstico

Ateu

Mórmon

Nenhum

Segunda filiação religiosa

Sim

Não

$63(87,5)$

Qual?

$1(14,3)$

Evangélica

$4(57,1)$

Espírita

$1(14,3)$

Umbandista

Outra

\section{Praticante}

Sim

Não

\section{Religiosidade organizacional (Durel)}

(Com que frequência você vai a uma igreja, templo ou outro encontro religioso?) $8(11,1)$

Mais de uma vez por semana

Uma vez por semana

Duas a três vezes por mês

Algumas vezes por ano

Uma vez por ano ou menos

Nunca

Religiosidade não organizacional (Durel)

(Com que frequência você dedica seu tempo a atividades religiosas individuais, como preces, rezas, meditações, leitura da Bíblia ou de outros

textos religiosos?)

Mais de uma vez ao dia

Diariamente

Duas ou mais vezes por semana

Uma vez por semana

Poucas vezes por mês

Raramente ou nunca

Religiosidade intrínseca (Durel)

a) Em minha vida, eu sinto a presença de Deus (ou do Espírito Santo).

Totalmente verdade pra mim

Em geral é verdade

Não estou certo

Em geral não é verdade

Não é verdade

b) Minhas crenças religiosas estão realmente por trás de toda a minha maneira de viver.

Totalmente verdade pra mim

Em geral é verdade

Não estou certo

Em geral não é verdade

Não é verdade

c) Eu me esforço muito para viver a minha religião em todos os aspectos da vida.

Totalmente verdade pra mim

Não estou certo

Em geral não é verdade

Não é verdade

Inventário de Religiosidade Intrínseca

(média dos escores, com variação de 1 a 5)

Você acredita em Deus ou em um "poder superior"?

Sim

Não desejo responder 


\section{TABELA 2}

Resultados do questionário sobre percepções do papel da espiritualidade no cuidado médico - resultados expressos como n (\%)

\begin{tabular}{|c|c|c|c|c|}
\hline & Mulheres & Homens & Total & $\mathbf{P}$ \\
\hline $\begin{array}{l}\text { Tratamento biológico/físico (medicações/cirurgias) } \\
\text { Muito importante } \\
\text { importante } \\
\text { Neutro } \\
\text { Pouco importante } \\
\text { Irrelevante }\end{array}$ & $\begin{array}{r}34(79,1) \\
8(18,6) \\
1(2,3) \\
-\end{array}$ & $\begin{array}{r}23(76,7) \\
6(20) \\
- \\
- \\
1(3,3)\end{array}$ & $\begin{array}{r}57(78,1) \\
14(19,2) \\
1(1,4) \\
- \\
1(1,3)\end{array}$ & 0.539 \\
\hline $\begin{array}{l}\text { Cuidado das questões sociais } \\
\text { Muito importante } \\
\text { Importante } \\
\text { Neutro } \\
\text { Pouco importante } \\
\text { Irrelevante }\end{array}$ & $\begin{array}{r}33(76,7) \\
9(20,9) \\
1(2,3) \\
- \\
-\end{array}$ & $\begin{array}{r}25(83,3) \\
5(16,7) \\
- \\
-\end{array}$ & $\begin{array}{r}58(79,5) \\
1419,2) \\
1(1,40) \\
- \\
-\end{array}$ & 0,618 \\
\hline $\begin{array}{l}\text { Cuidado das questões psicológicas } \\
\text { Muito importante } \\
\text { Importante } \\
\text { Neutro } \\
\text { Pouco importante } \\
\text { irrelevante }\end{array}$ & $\begin{array}{r}38(88,4) \\
5(11,6) \\
- \\
- \\
-\end{array}$ & $\begin{array}{r}24(80) \\
6(20) \\
\quad- \\
- \\
-\end{array}$ & $\begin{array}{r}62(84,9) \\
11(15,1) \\
- \\
- \\
-\end{array}$ & 0,342 \\
\hline $\begin{array}{l}\text { Cuidado das questões espirituais } \\
\text { Muito importante } \\
\text { Importante } \\
\text { Neutro } \\
\text { Pouco importante } \\
\text { irrelevante }\end{array}$ & $\begin{array}{r}22(51,2) \\
18(41,9) \\
3(7) \\
- \\
-\end{array}$ & $\begin{array}{r}14(46,7) \\
11(36,7) \\
3(10) \\
1(3,3) \\
1(3,3)\end{array}$ & $\begin{array}{r}36(49,3) \\
29(39,7) \\
6(8,2) \\
1(1,4) \\
1(1,4)\end{array}$ & 0,516 \\
\hline $\begin{array}{l}\text { A saúde espiritual contribui para a saúde física. } \\
\text { Concordo fortemente } \\
\text { Concordo } \\
\text { Neutro } \\
\text { Discordo } \\
\text { Discordo fortemente }\end{array}$ & $\begin{array}{r}23(53,3) \\
19(44,2) \\
1(2,3) \\
- \\
-\end{array}$ & $\begin{array}{r}15(50) \\
12(40) \\
3(10) \\
- \\
-\end{array}$ & $\begin{array}{r}38(52,1) \\
31(42,5) \\
4(5,5) \\
- \\
-\end{array}$ & 0,365 \\
\hline
\end{tabular}

A fé em alguma religião ou alguma forma de espiritualidade é um importante aspecto na vida de muitos pacientes.

Concordo fortemente

Concordo

Neutro

Discordo fortemente

Os pacientes geralmente querem que seus médicos tenham conhecimento de suas crenças, valores e necessidades religiosas/espirituais.

Concordo fortemente

Concordo

$\begin{array}{rrr}5(11,6) & 1(3,3) & 6(8,2) \\ 23(53,5) & 11(36,7) & 34(46,6) \\ 11(25,6) & 11(36,7) & 22(30,1) \\ 3(7) & 6(20) & 9(12,3) \\ 1(2,3) & 1(3,3) & 2(2,7)\end{array}$

Neutro

Discordo

$1(2,3)$

$1(3,3)$

Discordo fortemente

Profissionais de saúde devem dividir com os pacientes suas próprias crenças espirituais.

Concordo fortemente

Concordo

Neutro

Discordo

Discordo fortemente

$\begin{array}{rrr}- & - & - \\ 5(11,6) & 5(16,7) & 10(13,7) \\ 14(32,6) & 14(46,7) & 28(38,4) \\ 16(37,2) & 7(23,3) & 23(31,5) \\ 8(18,6) & 4(13,3) & 12(16,4)\end{array}$




\section{TABela 2}

Resultados do questionário sobre percepções do papel da espiritualidade no cuidado médico - resultados expressos como n (\%)

\begin{tabular}{|c|c|c|c|c|}
\hline & Mulheres & Homens & Total & $\mathbf{P}$ \\
\hline $\begin{array}{l}\text { Profissionais de saúde devem dividir suas crenças espirituais com os pacientes apenas } \\
\text { quando solicitados. } \\
\text { Concordo fortemente } \\
\text { Concordo } \\
\text { Neutro } \\
\text { Discordo } \\
\text { Discordo fortemente }\end{array}$ & $\begin{array}{r}3(7) \\
22(51,2) \\
13(30,2) \\
2(4,7) \\
3(7)\end{array}$ & $\begin{array}{r}4(13,3) \\
9(30) \\
12(40) \\
2(6,7) \\
3(10)\end{array}$ & $\begin{array}{r}7(9,6) \\
31(42,5) \\
25(34,2) \\
4(5,5) \\
6(8,2)\end{array}$ & 0,489 \\
\hline
\end{tabular}

Os médicos devem deixar o cuidado das questões espirituais aos capelães ou outros profissionais.

Concordo fortemente

$\begin{array}{rrr}1(2,3) & 1(3,3) & 2(2,7) \\ 5(11,6) & 5(16,7) & 10(13,7) \\ 12(27,9) & 10(33,3) & 22(30,1) \\ 20(46,5) & 9(30) & 29(39,7) \\ 5(11,6) & 5(16,7) & 10(13,7)\end{array}$

Neutro

$5(11,6)$

$5(16,7)$

Discordo

Discordo fortemente

\begin{tabular}{|c|c|c|c|c|}
\hline $\begin{array}{l}\text { A fé ou as crenças espirituais podem afetar a resposta clínica do paciente ao seu } \\
\text { diagnóstico } \\
\text { e ao prognóstico. } \\
\text { Concordo fortemente } \\
\text { Concordo } \\
\text { Neutro } \\
\text { Discordo } \\
\text { Discordo fortemente }\end{array}$ & $\begin{array}{r}14(32,6) \\
28(65,1) \\
1(2,3) \\
- \\
-\end{array}$ & $\begin{array}{r}10(33,3) \\
15(50) \\
3(10) \\
2(6,7) \\
-\end{array}$ & $\begin{array}{r}24(32,9) \\
43(58,9) \\
4(5,5) \\
2(2,7) \\
-\end{array}$ & 0,141 \\
\hline $\begin{array}{l}\text { Algumas vezes, os pacientes se recuperam de doenças por maneiras que não podem ser } \\
\text { explicadas cientificamente. } \\
\text { Concordo fortemente } \\
\text { Concordo } \\
\text { Neutro } \\
\text { Discordo } \\
\text { Discordo fortemente }\end{array}$ & $\begin{array}{r}12(27,9) \\
22(51,2) \\
7(16,3) \\
2(4,7) \\
-\end{array}$ & $\begin{array}{r}10(34,5) \\
12(41,4) \\
4(13,8) \\
1(3,4) \\
2(6,9)\end{array}$ & $\begin{array}{r}22(30,6) \\
34(47,2) \\
11(15,3) \\
3(4,2) \\
2(2,8)\end{array}$ & 0,449 \\
\hline $\begin{array}{l}\text { Quais das seguintes opções você considera as mais importantes que o médico promova } \\
\text { no cuidado espiritual do paciente? (até três escolhas) } \\
\text { Colher a história espiritual } \\
\text { Aconselhamento espiritual } \\
\text { Fazer contato com líderes religiosos do paciente } \\
\text { Sugerir leituras, músicas ou programas de TV religiosos } \\
\text { Fazer orações com o paciente } \\
\text { Nenhuma das alternativas } \\
\text { Outros }\end{array}$ & & & $\begin{array}{r}44(60,3) \\
28(38,4) \\
16(21,9) \\
12(16,4) \\
4(5,5) \\
9(12,3) \\
5(6,8)\end{array}$ & \\
\hline
\end{tabular}

\begin{tabular}{lr}
\multicolumn{1}{c}{ TABELA 3} \\
Resultados do questionário sobre R/E no ensino médico \\
Total \\
n (\%)
\end{tabular}

Abordar pesquisas científicas sobre o impacto da espiritualidade na saúde deve fazer parte do currículo médico na graduação. Concordo fortemente

Concordo

Neutro

Discordo

Discordo fortemente 
TABELA 3

Resultados do questionário sobre R/E no ensino médico

Ensino de questões espirituais é mais apropriado como um componente opcional da graduação médica para aqueles alunos que tenham mais interesse no tema.

Concordo fortemente

Concordo

Neutro

Discordo

Discordo fortemente

Ensino de questões espirituais deve ser incorporado ao currículo médico obrigatório para todos os estudantes.

Concordo fortemente

Concordo

Neutro

Discordo

$21(29,2)$

Discordo fortemente

Ensino de questões espirituais aos alunos de graduação médica deve ser ministrado por médicos.

Concordo fortemente

Concordo

Neutro

Discordo

$19(26,4)$

Discordo fortemente

Quais das seguintes opções poderiam ser utilizadas para o ensino de cuidados espirituais no currículo médico?

Assuntos escolhidos pelos alunos

Palestras/conferências com convidados

Seminários em pequenos grupos

Ensino prático junto aos pacientes

Acompanhamento do capelão hospitalar

Visitas a organizações/templos religiosos

Não deve ser ensinado

Como o aprendizado em espiritualidade deve ser avaliado?

Laboratório de habilidades

Portfólio Reflexivo

À beira do leito

Avaliação escrita

Não deve ser avaliado

prática e no ensino médicos. O estudo da $\mathrm{R} / \mathrm{E}$ dos médicos tem sido reconhecido como um tema importante, tendo em vista seu potencial de influenciar decisões médicas e mesmo a escolha dessa carreira ${ }^{16}$. O motivo de se avaliar o perfil da $\mathrm{R} / \mathrm{E}$ do médico é o reconhecimento da literatura internacional, que considera importante a experiência pessoal do estudante nas estratégias de ensino relacionadas à espiritualidade. Dessa forma, o ensino da $\mathrm{R} / \mathrm{E}$ é mais efetivo quando se vai além da teoria, permitindo a exposição de visões pessoais, receios, crenças, dificuldades da prática profissional em situações desafiantes, nas quais frequentemente emergem conteúdos de cunho religioso $^{7}$. Essa peculiaridade do ensino da R/E na saúde está em sintonia com a proposta didático-pedagógica da UNA-SUS/UFCSPA, que pressupõe uma aprendizagem ativa, fundamentada em saberes que o aluno traz de sua prática cotidiana, de suas experiências no trabalho e na vida ${ }^{14}$.

\begin{tabular}{|c|c|c|c|}
\hline \multicolumn{4}{|c|}{$\begin{array}{l}\text { Correlação entre religiosidade intrínseca (segundo } \\
\text { Inventário de Religiosidade Intrínseca) e atribuição } \\
\text { de importância à R/E no cuidado médico }\end{array}$} \\
\hline $\begin{array}{l}\text { Cuidado das questões } \\
\text { espirituais na prática } \\
\text { clínica }\end{array}$ & $\begin{array}{l}\text { RI Alta } \\
(\geq 4) \\
(\%)\end{array}$ & $\begin{array}{l}\text { RI Baixa } \\
(\leq 2) \\
(\%)\end{array}$ & $\begin{array}{l}\text { Person, } \\
\text { Qui-Quadrado } \\
0,585\end{array}$ \\
\hline Muito importante & 62,2 & 45,5 & \\
\hline Importante & 32,4 & 45,5 & \\
\hline Neutro & 2,7 & 9,1 & \\
\hline Pouco importante & 2,7 & 0 & \\
\hline
\end{tabular}

Os termos religiosidade e espiritualidade, que são constructos complexos e multifacetados e sobre os quais não há definições consensuais, não foram definidos a priori pelos autores. Isto permitiu que os respondedores utilizassem seus 
próprios conceitos para responder aos questionários, captando um universo mais próximo da realidade do profissional e de sua prática clínica, sem a necessidade, portanto, de um aprendizado prévio de conceitos.

A literatura tem apontado um religiosity gap, ou seja, médicos costumam ter índices de $\mathrm{R} / \mathrm{E}$ menores do que os de seus pacientes, criando, muitas vezes, dificuldades de empatia e prejuízo na relação médico-paciente ${ }^{16}$. Os dados encontrados confirmam em parte a literatura, especialmente nas taxas de filiação religiosa, frequência religiosa e crença em Deus. Num levantamento nacional representativo da população brasileira, dos 2.346 adultos pesquisados, apenas 5\% diziam-se sem religião ${ }^{17}$, ao passo que a taxa verificada nesse estudo foi de 10,1\% (incluindo os que se declararam ateus, agnósticos e os que não seguem nenhuma denominação religiosa). Nesse mesmo levantamento, verificou-se que $37,2 \%$ da população adulta brasileira frequentam algum templo religioso uma ou mais vezes na semana, ao passo que dos médicos desse estudo apenas $26,4 \%$ têm essa prática regular. Em relação à variável crença em Deus, o levantamento do World Values Survey ${ }^{18}$ de 2014 apontou que $98,4 \%$ da população adulta brasileira acreditam, enquanto entre os médicos desse levantamento encontrou-se redução dessa taxa para $86,3 \%$, superior, no entanto, aos $68,5 \%$ encontrados em levantamento com médicos nos Estados Unidos ${ }^{16}$.

Outro dado parece confirmar a literatura nacional: a taxa de pessoas que frequenta ou segue mais de uma religião. $\mathrm{Na}$ população geral brasileira ${ }^{17}, 10,4 \%$ dos entrevistados referiram seguir uma segunda religião, ao passo que entre os médicos o índice foi de $12,5 \%$. Considerando-se que os dados foram obtidos tendo por base $17,2 \%$ dos médicos matriculados no curso, é possível que haja um viés de seleção nos resultados encontrados e que a realidade seja de diferenças ainda mais significativas no perfil religioso dos médicos quando comparado ao da população adulta nacional.

A inclusão da R/E nos currículos médicos nacionais é incipiente e praticada pela minoria das universidades. Segundo Luccheti et al. ${ }^{8}$, num levantamento com 47,4\% das escolas médicas brasileiras, apenas 10,4\% dos cursos de graduação dispõem de disciplinas dedicadas ao ensino de espiritualidade e saúde. Dos médicos avaliados nesse estudo, apenas $13,7 \%$ referiram ter estudado o tema na graduação, fato condizente, portanto, com os achados de Luccheti. Não foi avaliado, no entanto, como se deu esse processo de ensino-aprendizagem. É interessante ressaltar que 70,2\% dos médicos pesquisados consideram importante a inclusão da R/E como temática de estudos na formação médica, dado coerente com a relevância que atribuem ao tema, pois $89 \%$ deles consideram importante ou muito importante o cuidado espiritual na prática médica, índice equivalente à relevância que atribuem a outras formas de cuidado clínico, como o biológico, psicológico e social.

Apesar do reconhecimento da relevância para a prática profissional, os médicos tendem a desejar esse tema apenas como um conteúdo eletivo na graduação. Essa também é a opinião da maior parte dos estudantes de Medicina de outros países, tendo em vista a possibilidade de abordagem dos aspectos espirituais dos pacientes em diversos momentos ao longo da formação, como em disciplinas de semiologia médica, cuidados paliativos e psiquiatria ${ }^{7}$. Embora uma pequena minoria dos alunos tenha sido exposta a disciplinas que ensinaram a respeito de $\mathrm{R} / \mathrm{E}$ na graduação médica, a relevância conferida à $\mathrm{R} / \mathrm{E}$ como um fator importante no cuidado médico não parece ter sido mediada pela religiosidade intrínseca dos estudantes, pois não houve correlação estatisticamente significativa entre essa variável e a atribuição de importância à $\mathrm{R} / \mathrm{E}$ na prática clínica $(p=0,585)$, conforme apresentado na Tabela 4 .

A importância da fé na vida dos pacientes parece ser uma variável considerada relevante por ambos os gêneros no atendimento médico. Entretanto, o gênero feminino concordou mais fortemente com essa afirmação de forma estatisticamente significativa. A opinião dos médicos estudados está de acordo com os dados da literatura, conforme o estudo de Stewart et $a l .{ }^{17}$, que, numa revisão de 49 artigos sobre o tema, encontrou que a fé é importante para muitos pacientes, em particular aqueles com doenças graves, que utilizam frequentemente a $\mathrm{R} / \mathrm{E}$ como estratégia de coping positivo ${ }^{17}$.

Os médicos avaliados parecem reconhecer a importância da espiritualidade para os pacientes e para a saúde, mas são menos fortemente propensos a considerar o seu papel como cuidadores dessa dimensão, pois quase a metade respondeu de forma neutra ou concorda com a afirmação de que os cuidados espirituais devem ser delegados a outros profissionais. Essa aparente dicotomia entre a importância atribuída ao tema $\mathrm{R} / \mathrm{E}$ e o fraco reconhecimento do seu papel como agentes provedores desse cuidado pode ser, ao menos em parte, explicada pela autopercepção de inabilidade na abordagem do tema, resultado da falta de experiências de ensino e de desenvolvimento de habilidades para lidar com um tema tão sensível como a R/E dos pacientes, conforme vemos na literatura ${ }^{18}$.

A coleta da história espiritual ganhou destaque como a habilidade mais importante a ser desenvolvida pelo médico $(60,3 \%)$ e a estratégia de ensino do portfólio reflexivo como a mais adequada $(22,2 \%)$ para a avaliação dos alunos no ensino do tema R/E e saúde, ambos em sintonia com os dados encontrados em revisões anteriores ${ }^{7}$. 
O presente estudo contém algumas limitações metodológicas, como o delineamento transversal, com informações coletadas online. Além disso, generalizações devem ser utilizadas com cautela em outros contextos, devido à relativa baixa taxa de resposta $(17,2 \%)$. Entretanto, essa taxa está dentro do esperado para levantamentos que utilizam metodologia eletrônica (e-mail). Além disso, as taxas de resposta de estudos realizados via internet são mais baixas do que as obtidas nos estudos realizados presencialmente, por correio ou telefone. A taxa de resposta em estudos que investigam pessoas de negócios, psiquiatras e oncologistas não costuma ultrapassar $20 \%$. Outra possível limitação do estudo é que pode ter selecionado pessoas mais emocionalmente conectadas ao tema, tanto a favor, como contra. Assim, aqueles mais religiosos/espiritualizados e aqueles que não são religiosos/espiritualizados podem ter se sentido mais inclinados a responder ao questionário ${ }^{19}$

\section{CONCLUSÕES}

A aceitação do tema R/E pelos médicos especializandos em Saúde da Família pela UNA-SUS/UFCSPA foi considerada bastante satisfatória, conforme evidenciado pela importância atribuída ao tema, por suas concepções acerca do impacto destas dimensões na saúde dos pacientes e pelo interesse demonstrado no assunto como um elemento curricular na formação profissional. Este fato é digno de nota, uma vez que as humanidades são frequentemente negligenciadas na educação médica, apesar do seu papel na construção da integralidade na atenção à saúde ${ }^{20}$ e também pelas baixas taxas de experiências prévias de ensino em $\mathrm{R} / \mathrm{E}$.

Como proposto pelas Diretrizes Curriculares Nacionais (DCN) do Curso de Graduação em Medicina, o médico deverá formar-se sob uma ótica humanista, crítica e reflexiva e estar apto a corresponder às demandas da saúde da população de forma integral. Desse modo, a atenção para a saúde espiritual surge como fundamento essencial ${ }^{20}$. A carência de atividades teórico-práticas que ensejem uma qualificação profissional mínima para a abordagem da espiritualidade dos pacientes reitera a importância de buscar alternativas educacionais que proponham a construção de conhecimentos aplicáveis à prática clínica do médico brasileiro.

Dada a relevância do tema $\mathrm{R} / \mathrm{E}$ e suas demonstradas repercussões no âmbito da saúde física e mental, urge que a comunidade docente e discente nacional desenvolva propostas pedagógicas inovadoras, metodologicamente eficazes e apropriadas ao contexto da atuação médica no Sistema Único de Saúde, tanto na graduação, quanto nos programas de pós-graduação.

\section{REFERÊNCIAS}

1. Moreira-Almeida A, Lotufo Neto F, Koenig HG. Religiousness and mental health: a review. Rev Bras Psiquiatr. 2006;28:242-50.

2. Penna Guimarães H, Avezum A. O impacto da espiritualidade na saúde física.RevPsiq Clín. 2007;34 supl 1:88-94.

3. Lucchetti G, Lucchetti AL, Koenig HG. Impact of spirituality/religiosity on mortality: comparison with other health interventions. Explore (NY). 2011;7:234-8.

4. Curlin FA, Lantos JD, Roach CJ, Sellergren SA, Chin MH. Religious characteristics of U.S. physicians: a national survey. J Gen Intern Med. 2005;20:629-34.

5. Koenig HG, Hooten EG, Lindsay-Calkins E, Meador KG. Spirituality in medical school curricula: findings from a national survey. Int J Psychiatry Med. 2010;40:391-8.

6. Neely D, Minford EJ. Current status of teaching on spirituality in UK medical schools. Med Educ. 2008;42:176-82.

7. Dalla Colletta de Aguiar PR, Cazella SC, Costa MRC. What do we know about the teaching of religiosity/spirituality in medical undergraduate curricula? An integrative review, MedEdPublish, 2016;5:20.

8. Lucchetti G, Lucchetti AL, Espinha DC, de Oliveira LR, Leite JR, Koenig HG. Spirituality and health in the curricula of medical schools in Brazil. BMC Med Educ. 2012;12:78.

9. Lucchetti G, de Oliveira LR, Koenig HG, Leite JR, Lucchetti AL; SBRAME Collaborators. Medical students, spirituality and religiosity--results from the multicenter study SBRAME. BMC Med Educ. 2013;13:162.

10. Lucchetti G, Bassi RM, Lucchetti AL. Taking spiritual history in clinical practice: a systematic review of instruments. Explore (NY). 2013;9:159-70.

11. Fang ML, Sixsmith J, Sinclair S, Horst G. A knowledge synthesis of culturally- and spiritually-sensitive end-of-life care: findings from a scoping review. BMC Geriatr. 2016;16:107.

12. Lucchetti G, Granero AL. Spirituality and health's most productive researchers: the role of primary care physicians. Fam Med. 2010;42:656-7.

13. Ellman MS, Schulman-Green D, Blatt L, Asher S, Viveiros D, Clark J, Bia M. Using online learning and interactive simulation to teach spiritual and cultural aspects of palliative care to interprofessional students. J Palliat Med. 2012;15:1240-7. http://www.unasus.gov.br/page/una-sus/rede-una-sus. Acessado em 28 de dezembro de 2016.

14. Bell D, Harbinson M, Toman G, Crawford V, Cunningham $H$. Wholeness of healing: an innovative Student-Selected Component introducing United Kingdom medical students to the spiritual dimension in healthcare. South Med J. 2010;103:1204-9. 
15. Robinson KA, Cheng MR, Hansen PD, Gray RJ. Religious and spiritual beliefs of physicians J Relig Health. 2017;56:205.

16. Stewart WC, Adams MP, Stewart JA, Nelson LA. Review of clinical medicine and religious practice. J Relig Health. 2013;52:91-106.

17. Ellis MR, Campbell JD, Detwiler-Breidenbach A, Hubbard DK. What do family physicians think about spirituality in clinical practice? J FamPract. 2002;51:249-54.

18. Menegatti-Chequini MC, Gonçalves JP, Leão FC, Peres MF, Vallada H. A preliminary survey on the religious profile of Brazilian psychiatrists and their approach to patients' religiosity in clinical practice. BJPsych Open. 2016;2:346-352.

19. Ferreira AGC, Oliveira JAC, Jordán APW.Educação em Saúde e Espiritualidade: uma proposta de transversalidade na perspectiva do estudante. InterdisciplinaryJournalof Health Education2016;1:3-12.

\section{CONTRIBUIÇÃO DOS AUTORES}

Paulo Rogério Aguiar: elaboração do projeto, coleta, análise, interpretação dos dados, revisão teórica e discussão. Silvio César Cazella: orientação da pesquisa, análise dos dados e elaboração do projeto. Márcia Rosa da Costa: orientação da pesquisa, coleta de dados, elaboração do projeto, submissão ao CEP e aprovação final.

\section{CONFLITO DE INTERESSES}

Os autores declaram não haver conflito de interesses.

\section{ENDEREÇO PARA CORRESPONDÊNCIA}

Paulo Rogério Aguiar

Universidade Federal de Ciências da Saúde de Porto Alegre

Rua Sarmento Leite, 245

Centro Histórico - Porto Alegre

CEP 90050-170 - RS

E-mail: dallaguiar@gmail.com 